\title{
A Clustering Approach for Multiband Neighbor Discovery on 60 GHz WLAN
}

\author{
Davi da S. Brilhante $\mathbb{D}^{\mathrm{D}}$ and José F. de Rezende \\ Laboratory LAND, Department of Computing and System Engineering (PESC), Universidade Federal do Rio de Janeiro (UFRJ), \\ Rio de Janeiro, RJ, Brazil \\ Correspondence should be addressed to Davi da S. Brilhante; dbrilhante@land.ufrj.br
}

Received 1 November 2018; Revised 28 March 2019; Accepted 21 April 2019; Published 16 June 2019

Academic Editor: Lin Chen

Copyright (C) 2019 Davi da S. Brilhante and José F. de Rezende. This is an open access article distributed under the Creative Commons Attribution License, which permits unrestricted use, distribution, and reproduction in any medium, provided the original work is properly cited.

\begin{abstract}
The $60 \mathrm{GHz}$ mmWave unlicensed band has a very large spectrum available, divided into four orthogonal channels, which allows up to $7 \mathrm{Gbps}$ data rate. On the other side, the propagation in the $60 \mathrm{GHz}$ band is subject to severe path loss attenuation, which can be mitigated using highly directional antennas. This high directionality brings a new challenge to neighbor discovery; the devices now need to know the exact neighbors' physical location to successfully communicate with them. In order to expedite the neighbor discovery process, multiband protocols have been proposed in the literature in which a separate band is used for the exchange of control messages in an omnidirectional mode. Nonetheless, these proposals suffer from the control channel bottleneck problem due to the numerous messages that need to be exchanged in this channel. In this work, we propose a scheme that divides the network nodes in clusters and for each cluster we allocate one separate control channel and also a separate mmWave channel for beamforming only. The former separation allows decreasing the number of control messages exchanged in each control channel, and the latter allows the simultaneously execution of multiple beamforming. In conjunction to this clustering scheme, we propose a multiband protocol in which only the cluster leader performs beamforming and uses the control channel to propagate the information obtained during this process. We compare the existing protocols with our proposed clustering protocol in terms of average transmission time, overhead, and accuracy of neighbor discovery information.
\end{abstract}

\section{Introduction}

The mmWave bands have received lot of attention in the 5G umbrella. The huge spectrum availability in these bands makes it possible to have channels with larger bandwidths, and hence higher capacity. However, the signal propagation in these bands is hampered by severe path loss attenuation [1]. Therefore, in order to compensate this problem the use of highly directional antennas is of paramount importance. Hence, the communication between two nodes in these bands only takes place after they have beamformed to each other.

In the case of a network composed by multiple nodes, the entire process of all nodes performing a beamforming with all others is sometimes called neighbor discovery. In the case of network bootstrapping, this mechanism is also denominated initial access since it has to take place before any communication is possible. It can be naively performed by each node executing an exhaustive beamforming with each of its neighbors. However, this process can be very cumbersome and many works have proposed new mechanisms to speed up this process [2]. In addition, when multiple stations attempt to perform beamforming many collisions occur, which require a random exponential backoff technique before reattempting the process, significantly increasing the bootstrapping time.

For example, consider a network with an access point (AP) and a station (STA), both equipped with $m$ sectors directional antennas, trying to discover each other at mmWave band. Assuming that only these two nodes will send beamforming packets, that there are no collisions, and that they are both synchronized, $m^{2}$ time slots are necessary for the AP to send beamforming packets to the STA to complete one way of the beamforming process. To complete the beamforming, the STA will transmit in each beam of its antenna while the AP listens at the best beam recently discovered; thus more $m$ slots are spent. Finally, $m^{2}+m$ time slots are necessary for 
each STA completely beamforms with the AP [3], delaying the beginning of data packets transmission. In addition, in a scenario with more than one STA, the coordination of beamforming is critical to avoid collisions since directionality makes STAs deaf.

One class of neighbor discovery mechanisms encourages the use of a control channel, operating in a band different from the mmWave band, to organize this process. These mechanisms normally devise a protocol, called a multiband protocol, that defines the exchange of control messages in a channel that can be used omnidirectionally, such as in the 2.4 and $5 \mathrm{GHz}$ bands. In the work in [4], the mmWave nodes use a WiFi interface to exchange control messages at $2.4 \mathrm{GHz}$ band to define the order in which the nodes will perform the beamforming, and so avoiding collisions among them. Despite the large time reduction on the neighbor discovery offered by this kind of solution, it normally suffers from a known problem called control channel bottleneck due to the numerous messages that need to be exchanged in this channel.

In this work, we propose a multiband-based mechanism that divides the nodes in clusters and elects one of the nodes of the cluster to be a leader. Next, each cluster leader performs sequentially a beamforming with all the other nodes in the cluster. It then propagates the information obtained in this process to all nodes inside the cluster and all other cluster leaders. Through this information, all nodes can have a very good estimate of their neighboring location without the need of performing a complete beamforming with them. Besides, the clusterization allows parallelizing the beamforming of the leaders within the cluster. Results show that this protocol decreases the average time spent to a total network discovery around $85 \%$.

The remainder of this paper is organized as follows. Section 2 will present the related work. Section 3 presents the proposed neighbor discovery protocol as soon as a mathematical analysis of the error due to the suppression of the beamforming among all nodes. Numerical results are shown in Section 4. Finally, Section 5 brings authors' final considerations and future works.

\section{Related Works}

The emerging millimeter waves (mmWave) technology has called the attention of academia and industry due to its capacity to improve many aspects of mobile communications. Being quoted as a key technology to $5 \mathrm{G}$ mobile networks $[1,5,6]$, the huge bandwidth available at mmWave band can address the constraints of data rate and delay ambitioned by the Group Special Mobile Association (GSMA). Though the performance improvement that stems from this high spectrum availability, the propagation conditions are a critical point in using mmWave band. The path loss is handled using directional antennas [7]. By virtue of directionality, the simplistic omnidirectional approach to neighbor discovery is potentially not an efficient solution due to the small range that control signals can be received at such high frequencies. These conditions aforementioned highlight the necessity of efficient neighbor discovery mechanisms that lead to early detection of neighbor devices and their respective locations [8].
The IEEE 802.11ad standard [9] defines the Sector Sweep (SSW) process, which is the primary form of neighbor discovery. After performing a SSW, the node can improve the coarse information obtained by the Beam Refinement Protocol (BRP). During SSW, an initiator node starts a beamforming with another node, called responder. In this process, the initiator transmits directionally, in each sector of its antenna system, while the responder receives these frames in quasi-omnidirectional mode. This can be repeated in the opposite way, from responder to initiator, but with responder sending frames quasi-omnidirectionally and initiator receiving directionally [10]. The BRP phase provides a better antenna alignment resulting from antenna codebook exchange. Refining the beamforming results in higher data rate than only performing SSW.

The efficiency of neighbor discovery mechanisms was evaluated in some works [4, 11]. In [11], An et al. evaluated mathematically and through extensive simulations basic neighbor discovery mechanisms. Firstly, two different directional configurations, Directional-Omnidirectional (DO) and Directional-Directional (DD), were evaluated. They show that, despite the gain asymmetry of DO configuration, this configuration allows discovering the neighbors fastest than DD configuration. Second, the authors evaluate the performance of one-way and Handshake-based beamforming and conclude that in a multiuser scenario a one-way configuration achieves better performance than Handshake-based due to the overhead caused by directional acknowledgment messages.

Mathematical analyses are also conducted in [8], but authors consider the primary reflections influence over neighbor discovery process. Two neighbor discovery approaches were tested, Direct Discovery and Gossip-based discovery. The results reveal that gossip-based discovery is faster than direct discovery due to the contribution of indirect discovery when nodes gossip about their neighbors. Even in scenarios with furniture or walls, gossip-based discovery presents a better performance. On the other hand, it is difficult to state about the influence of obstacles on neighbor discovery because they not only block the signals but also establish new links via reflected paths.

There are also some works in the literature related to clustering in mmWaves. Some approaches, such as the IEEE 802.11 ad amendment $[12,13]$, apply clustering in the management, power control and cochannel interference reduction in scenarios with a dense deployment of base stations, for example, by scheduling transmissions to avoid collision inside a cluster and controlling transmission power. In [14], a mixed integer linear programming problem is devised to cluster femto access points (FAPs) and femto users (FUs) in a way to increase the number of LoS links and reduce interference. $X u$ et al. in [15] propose an agglomerative algorithm that clusters the users in groups to schedule beamforming in a base station (BS), iteratively merging users into groups to provide high spectral efficiency. Although the method described in [15] does not provide any schedule of transmissions and as only one user in each will communicate with the BS, this system is prone to energy fault considering the user equipment power constraints. Dense scenarios of wearable 
devices are investigated in [16], in which authors conclude that clustering protocols are potential feasible solutions to mmWave constraints imposed to MAC layer. In our paper, clustering is applied in the exploitation of multiple channels where clusters are distributed and the nodes can exchange control information to accelerate neighbor discovery time and, once the control phase is finished, neither cluster nor multiple channels are used to transmit and receive data.

Regarding the multiband approach, an RTS/CTS (Request to Send/Clear to Send) handshake is used in [17] to make nodes aware of incoming transmissions, not only the intended receiver but also the nodes within the transmission range. This strategy has significantly reduced deafness and collisions on mmWave WLANs, and hence improved the network capacity, but it does not address the problem of discovery. Concerning the neighbor discovery by using a multiband approach, the MDND (Multiband Directional Neighbor Discovery) mechanism [4] describes a control protocol that allows a collision-free neighbor discovery under reservation of the mmWave channel. The control messages collect devices' features and channel availability and signal the devices to change to the mmWave channel. In this protocol, the devices already associated with the access point (AP) that desire to transmit should request a list of devices and their capabilities. Once the devices possess this list, subsequent messages inform the modulation and coding scheme, the transmission duration, and the allocation of service period and finally indicate to the nodes that they have to change to the mmWave channel to perform the beamforming. Therefore, MDND avoids deafness and mmWave channel fluctuations on control messages transmissions but, on the other hand, these control messages can affect the discovery time due to the numerous access necessary to provide a two nodes discovery. Authors also conduct a mathematical analysis of expected discovery time and energy consumption on the discovery, which are validated by simulations.

In our previous work [18], two neighbor discovery protocols, which also implemented multiband features, were studied and exhaustively tested in terms of neighbor discovery accuracy and total discovery time. These protocols are based on a central node that discovers the other ones. In the first protocol, MuNDi MaPP, the central node sequentially beamforms with all other nodes, creates a map of neighbors with all gathered information, and shares this map with all other nodes. Conversely, in the second proposed protocol, instead of propagating the map of neighbors, a gossip technique is applied, given to each node the probability to overhear the beamforming of its neighbors and then discover opportunistically the nodes around by capturing beamforming frames.

In the novel approach presented in this work, the network is divided into clusters. For each cluster, one node is designated as leader, which is in charge of gathering discovery data inside a cluster. To each cluster, a control and a beamforming channel are designated. Afterwards, once all clusters finish gathering the neighbor map, the cluster leaders exchange their maps among themselves. The main contribution of this proposed algorithm is to reduce the neighbor discovery time by parallelizing the beamforming through the network clustering. Furthermore, the proposed algorithm reduces the control overhead imposed by the neighbor discovery phase in relation to MDND [4], as will be further presented in Section 4.2.

\section{CMPND: Clustering Multiband Protocol for Neighbor Discovery}

This section presents the proposed mechanism for neighbor discovery based on the multiband approach, called herein CMPND. Despite the use of multiband schemes which leads to the reduction of spatial gain and asymmetry in range caused by omnidirectional communications [19], a good design of the multiband protocol can mitigate these problems and improve the performance when compared to singleband approaches. Hence, the main goals of CMPND are to reduce the number of messages exchanged in the control band in order to minimize the contention in the control channel, decrease the time to each node in the network to discover all other nodes trough beamforming parallelization, and finally deliver high throughput data transmissions in mmWave band.

With these goals in mind, the network is coordinated by a central node and the nodes are divided into clusters. Within each cluster, only the leader of the cluster performs the beamforming with the other nodes in an organized way. After that, the cluster leader propagates the obtained information to all other nodes in the cluster and to the other cluster leaders. By using this information, all nodes in the network can estimate with a very high probability the location of the other nodes. In the next subsection, we will describe in detail the CMPND protocol. Following, we will present the information acquired during beamforming and the calculation used to estimate the position of the nodes. Next, we provide a mathematical analysis of the probability error incurred in these calculations as function of the errors in the estimate of the distance and angle of arrival.

3.1. CMPND Protocol. Before explaining the details of the proposed protocol, we introduce the assumptions under which it was designed. First, the neighbor discovery in the context of networks using directional antennas consists in building the knowledge about all neighbors' location in terms of the best beam (or angle) to be used to communicate with each of them individually [20]. The best beam is the one with which the node communicates with the largest rate, i.e., the largest SINR (Signal-to-interference-plus-noise ratio), with that neighbor. In addition, we consider that the nodes are not equipped with GPS and/or are operating in an indoor environment. Concerning the control channel, we assume that it is provided by an off-the-shelf WiFi radio operating with an omnidirectional antenna and the nodes can operate in different control channels that do not overlap among them. There is a previously configured channel at the control band, called rendezvous channel, which is used to carry out the initial stages of the protocol. In what concerns the mmWave band, we also assume that the nodes can operate in different channels and there is also a common channel, called intercluster channel. In addition, we consider that the network is single-hop at both operating bands. 
Let a wireless network be composed by $K$ stationary nodes equipped with two interfaces operating in different bands, one at millimeter waves and the other at a legacy band for the data and control planes, respectively. First, at the network bootstrapping, every operation is done at the control band, using the rendezvous channel. As soon as the nodes are powered up, they begin a process to elect a main leader which will coordinate the clustering process. Each node is initially a candidate to be the main leader and it broadcasts messages at the rendezvous channel. The algorithm to elect a leader in an asynchronous and distributed scenario is described in [21], with worst case time complexity $O(K)$ and message complexity $O(K \log K)$.

The $K$ nodes are divided into $C$ clusters and for each cluster one of the nodes plays the role of the cluster leader. The other $K-C$ nodes, called regular nodes, are aware of the cluster leader and have to be associated with it. The clusters will have the same number of components as possible. For example, a network with $K$ nodes and 3 clusters would have approximately $K / 3$ nodes in each cluster. Maintaining the clusters roughly with the same number of nodes guarantees a minimal time to each cluster to perform the whole operation. The clustering process is initially performed by the main leader, which allocates nodes to the clusters according to some criteria, designates the leader of each cluster, and assigns one orthogonal channel in both bands (mmWave and $\mathrm{WiFi}$ ) to each cluster. Therefore, the nodes within each cluster can exchange frames during the discovery process in parallel with nodes of the other clusters without interference between them. We called this preliminary stage of bootstrapping phase, depicted in Figure 1.

The clustering algorithm executed by the main leader is presented in Algorithm 1. The algorithm is a greedy heuristic to group nodes based on its location and keep the number of elements in each cluster uniform. We consider that, during the election process, the RSSI (Received Signal Strength Indication) of each message is stored by the main leader, what gives origin to a distance matrix. Then, the main leader orders the distances (line 10), pair by pair, and chooses to be cluster leader to the ones which are nearest to his neighbors (line 14). After choosing the cluster leaders, the nearest nodes to the cluster leaders are designated to be in the cluster respective to the leader (line 17). Finally, if there are any remaining nodes, once the quotient between the number of nodes and number of channel is not always integer in a real scenario, they are all allocated to the nearest cluster which has not received no other remaining node (line 21).

After all nodes are associated with its respective cluster leader, it distributes an ordering number to each node within the cluster with the aim of scheduling the beamforming among them. For that, it uses the following tuple: $\left[\left(i d_{1}, s_{1}\right),\left(i d_{2}, s_{2}\right), \ldots,\left(i d_{k-1}, s_{k-1}\right)\right]$, where $i d_{i}$ uniquely identifies the node $i$ and $s_{i}$ is the number that identifies the beamforming slot to be used by this node while all others stay in idle state. These messages of beamforming scheduling are sent in unicast to each other node at the control channel reserved for each cluster. Then, it starts the beamforming phase in mmWave designated channel during which the cluster leader performs an exhaustive search of each node in the cluster in its respective slot. Thus, each cluster leader will perform $(K-C) / C$ beamforming.

At the end of the beamforming phase, each cluster leader has built knowledge about the nodes in the cluster, which we call a cluster map. The cluster map contains the identity $I d_{i}$ of node $i$, the angle of arrival $\alpha_{i}$ used to receive from this node, and the estimated distance $d_{i}$ to this node. Some considerations about these last two values will be done in the next subsection. Next, the cluster leaders perform beamforming among them at the intercluster channel and transmit, piggybacked on the beamforming frames, the cluster map that each cluster leader has built. Let us assume that cluster leader $A$ has successfully received the map from cluster leader $B$ and has terminated the beamforming. Now, cluster leader $A$ knows the angle $\theta_{A B}$ to transmit to the cluster leader $B$. By the time that the cluster leader $A$ possesses the cluster map of $B$ and the angle $\theta_{A B}$, it is also able to transmit to any node that belongs to cluster $B$. When all cluster leaders are finally beamformed with each other, they have a complete view of the network with the corresponding global map. This global knowledge is now passed to regular nodes that belong to their cluster. Notice that all these information is passed through the respective control channel, except the information among cluster leaders that is passed through the mmWave band. Figure 2 shows the different phases of the protocol from the cluster formation (Figure 2(a)) to the submission of the global map to the regular nodes (Figure 2(d)).

At the end of global map broadcasting, each node has an estimate of the position of all other nodes. Before transmitting to a receiver node, the transmitter performs a RTS/CTS exchange with the receiver at rendezvous channel followed by a beamforming refinement, which allows identifying whether the target beam encountered using the propagated global map is correct, and otherwise, to find the right one. The refinement is performed by sending a refinement packet to a larger beam (refining beam) that contains the target beam in the middle. In case of a positive feedback from the receiver, the transmitter can now find the specific beam by partitioning this larger beam into multiple smaller subbeams and sweep through them. The phase of transmissions is detailed in Figure 3. The larger is the first beam, the slower is this process of refinement. Losses of refinement packets trigger the search of the receiver in the adjacent beams of the refining beam. Therefore, the more accurate is the information obtained by the map, the faster is the refinement phase.

Since the map of another cluster is first recalculated by the cluster leader, the error in transmission angle calculation is potentially bigger than the angles calculated inside the cluster. Let us suppose a set of clusters $A$ and $B$. The leaders of $A$ and $B$ are beamformed and have already exchanged the maps of the clusters they belong to. These maps will be recalculated by them and subsequently broadcasted inside their respective clusters. Thus, the transmission angles are first calculated by the leader of $B$ inside the cluster. Then, the map of $B$ is transmitted and recalculated by the leader of $A$, inserting errors to the angle. Finally, the leader of $A$ transmits the map of cluster $B$ to the regular nodes, which adds more errors to the angles. Hence, the clustering scheme incurs in less accuracy of angle transmissions than a direct approach. 


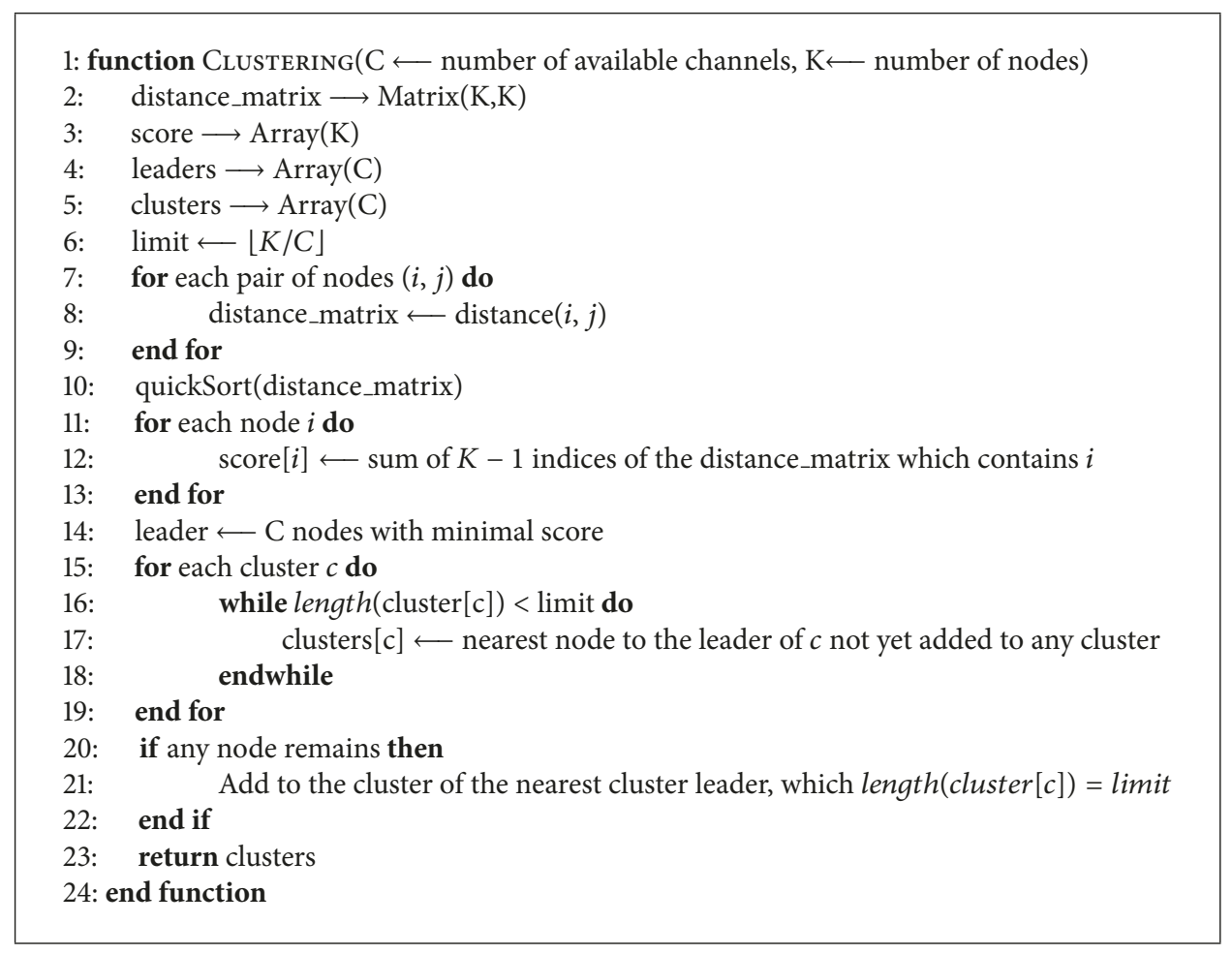

Algorithm 1: Clustering greedy algorithm.

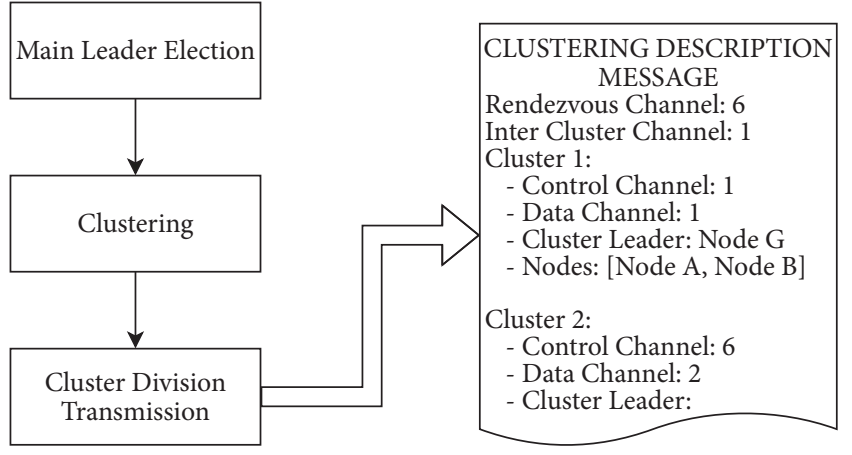

FIGURE 1: CMPND protocol bootstrapping phase.

Notwithstanding the worsening of accuracy, the clustering approach is more efficient, due to its parallelization, as further observed in Section 4.1.

In the next subsection, we will describe the information which is obtained during the beamforming and how this information is used by a node to calculate the position of the other nodes even without performing a beamforming with them.

3.2. Transmission Angle Calculation. As mentioned above, angle and distance are the main information to carry out any transmission that relies on the map propagated by cluster leader. This section explains the properties that permit a precise distance measurement and the subjacent calculus of angle estimation.

The estimation of the distance among two nodes with a high accuracy is possible in the mmWave band due to low scattering characteristics in this region of spectrum. In $[22,23]$, indoor localization mechanisms are proposed based on the exploitation of limited scattering, achieving submeter precision in distance measurement with high probability. Maletic et al. [24] obtain precision enhancements in real experiments which result in $16 \mathrm{~cm}$ error in distance measurements. Also, estimating the maximum direction of angle of arrival (AoA) is feasible with $\pm 5^{\circ}$ of precision, according to [25], and is more reliable than Time Difference of Arrival (TDoA) and Received Signal Strength (RSS), as evaluated in [26].

Consider the nodes $i$ and $j$ and the cluster leader $L$, as shown in the Figure 4. As previously said, during the beamforming phase, the cluster leader estimates both the distances $d_{i}$ and $d_{j}$ and the transmission angles $\alpha_{i}$ and $\alpha_{j}$ for each node, respectively. In a Cartesian complex representation, with origin in the cluster leader $L$ position, $Z_{i}$ and $Z_{j}$ are the complex coordinates of nodes $i$ and $j$, respectively. They are given by

$$
\begin{aligned}
& Z_{i}=d_{i} e^{j \alpha_{i}} \\
& Z_{j}=d_{j} e^{j \alpha_{j}}
\end{aligned}
$$

By propagating the distance $d_{i}$ and angle $\alpha_{i}$ inside the cluster, the nodes $i$ and $j$ can calculate their respective transmission angles to communicate between them. In the following, we show how $\theta_{i j}$, i.e., the transmission angle from the node $i$ to the node $j$, can be calculated with or without a common orientation between them. The $\theta_{j i}$ can be calculated in a similar way. 


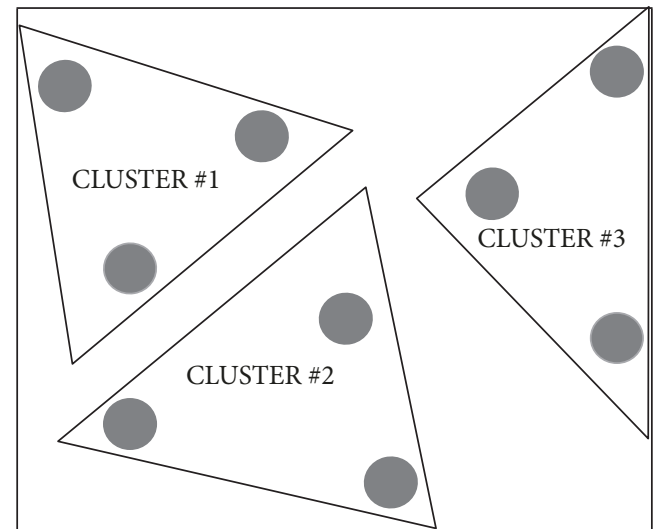

(a) Clustering

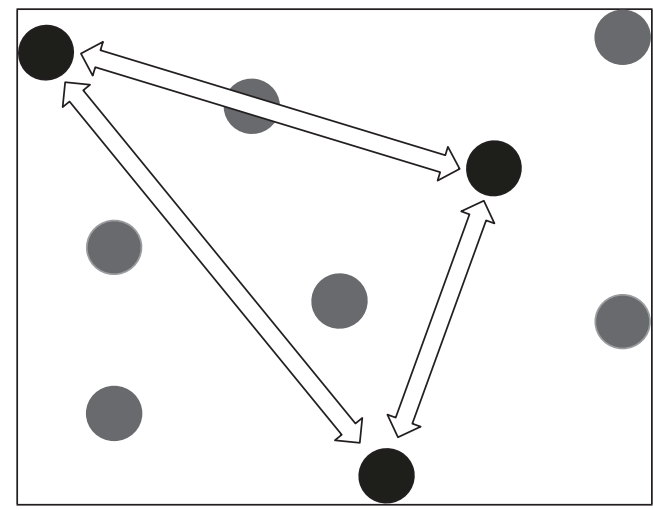

(c) Beamforming among cluster leaders with the propagation of local maps

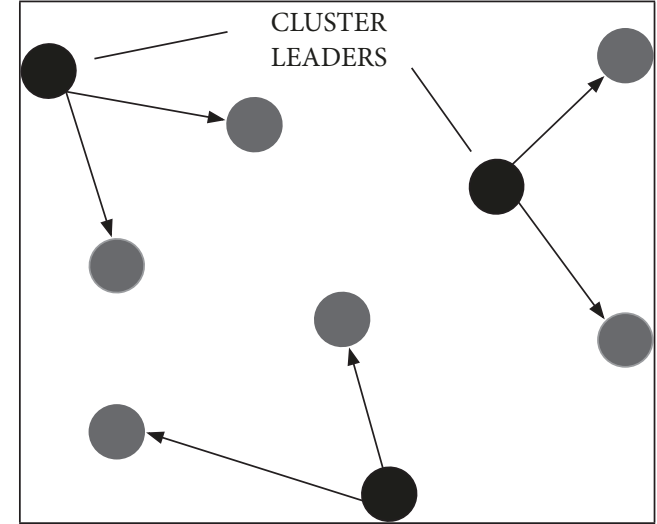

(b) Beamforming inside the cluster

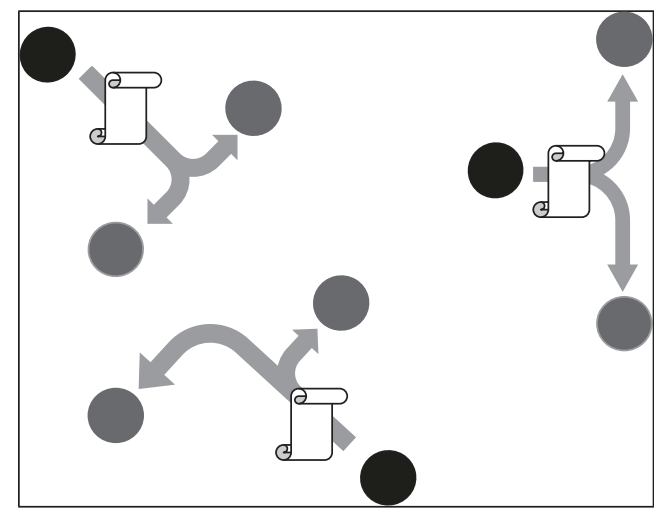

(d) Propagation of the global map inside the cluster

FIGURE 2: CMPND clustering subphases.

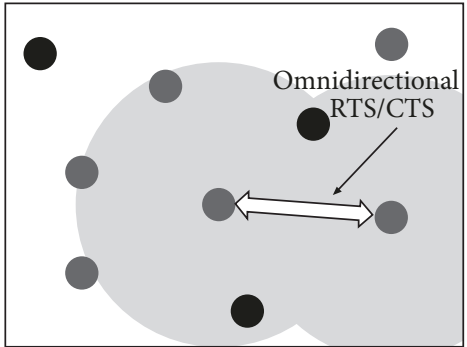

(a) RTS/CTS exchange at the control band

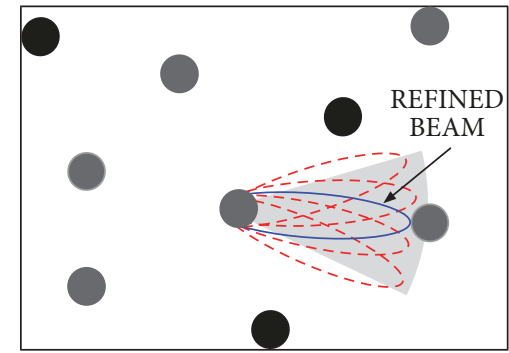

(b) Beam refinement between transmitter and receiver

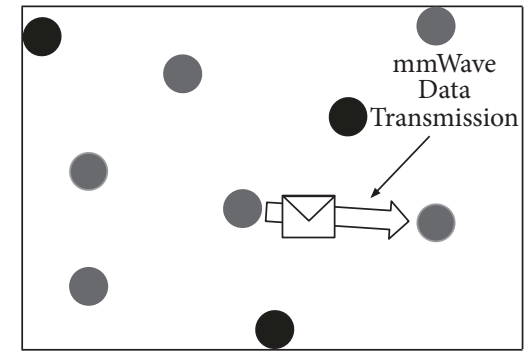

(c) Data transmission at mmWave band with high throughput

FIGURE 3: CMPND transmission phase.

$$
\theta_{i j}=\arg \left(Z_{i}-Z_{j}\right)
$$

where $\arg (\cdot)$ stands for the complex argument operation, which results in the angle between the positive real axis of the complex Cartesian plane and the line segment that bounds a complex point to the origin.

As shown above, by propagating the information collected during the beamforming, i.e., the cluster map, the leader allows each node inside the cluster to calculate its transmission angle to all the other nodes. Also, when a cluster leader $L$ of the cluster $C$ propagates the information to the other cluster leaders, they can also calculate their transmission angles to the nodes of the cluster $C$. They just have to estimate their distance and transmission angles to the leader $L$, which is the information that they acquire when they do the beamforming among them.

Until now, we have used the transmission angle $\theta_{i j}$ to define the position of a neighbor node $j$ relative to the node $i$. However, in the beamforming process, these angles are normally mapped to beam numbers, i.e., a node knows its neighbors positions by the number of the beam that it has to use to communicate with them. The estimation of the angle 


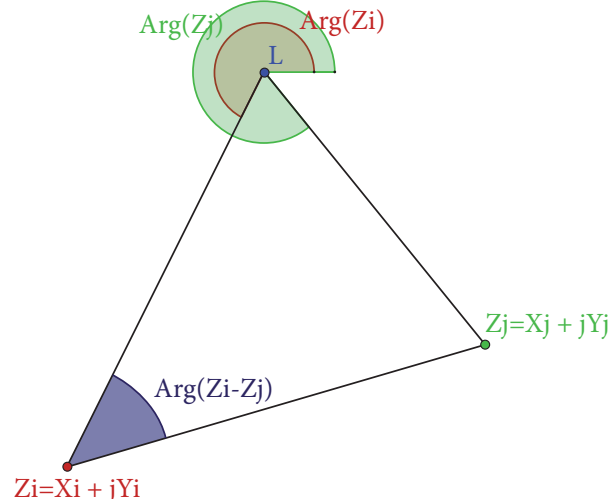

Figure 4: Placement of nodes $i, j$ and their angles related to leader node $L$.

is based on the result of the beamforming among the two nodes. After the beamforming, the receiver node informs the transmitter which beam of the latter has achieved the greatest signal-to-noise ratio (SNR). Based on that, the transmitter uses the middle of the beam as the angle to be used to reach the receiver node. This approximation adds an error to the process.

In the next subsection, we will derive the error probability in the correct estimation of these angles when the estimates of the distance and the transmission angles are not correct. Also, we derive the error probability when these angles are converted to beam numbers.

3.3. Understanding the Nodes Positioning Error Probability. As aforementioned, the CMPND efficiency on providing the right positioning of the nodes depends on angle and distance estimated by the beamforming process. Nevertheless, the nodes are not able to acquire this information with a total accuracy. In an ideal scenario, (3) gives a model of the system. Now, we will model the system through a continuous random variable $D_{i}$, whose random event is the distance estimate from leader to node $i$, in order to take into account errors during these measurements. Thus, (3), which determines the transmission angle from node $i$ to node $j$, may be rewritten as

$$
T=\frac{D_{i} / D_{j}}{\sin \left(\alpha_{i j}\right)}-\operatorname{cotan}\left(\alpha_{i j}\right)
$$

$T$ is a continuous random variable that expresses the cotangent of $\theta_{i j}$, which is the cotangent of the transmission angle of $i$ to $j$. The $\alpha_{i j}$ represents the angle formed between these two nodes from the point of view of the leader $L$ and it is equal to $\alpha_{i}-\alpha_{j}$. Assume that $D_{i}$ and $D_{j}$ are random variables with probability density functions $f_{D}(d)$, according to the uniform distribution given by (5)

$$
f_{D}(d)= \begin{cases}\frac{1}{2 \varepsilon}, & \text { if } \widehat{d}-\varepsilon \widehat{d} \leq d \leq \widehat{d}+\varepsilon \widehat{d} \\ 0, & \text { otherwise }\end{cases}
$$

where $\hat{d}$ is the real distance without errors between the leader and a node. The constant $\varepsilon$ represents the error in the measurement of the distance during the beamforming and $\varepsilon \in[0,1]$. To alleviate the notation, we will denote (4) by

$$
T=\frac{Z}{\sin \left(\alpha_{i j}\right)}-\operatorname{cotan}\left(\alpha_{i j}\right)
$$

$Z$ is the quotient of two independent random variables, $D_{i}$ and $D_{j} \cdot \sin \left(\alpha_{i j}\right)$ and $\operatorname{cotan}\left(\alpha_{i j}\right)$ are constants, respectively, a scale shift and a translation. Being both linear operations, the transformation of random variable $Z$ in $T$ is mathematically simple.

The probability distribution functions and cumulative distribution function of quotient of two independent uniform distributions $Z$ could be calculated by the law of total probability in the case of continuous random variables.

As previously said, the transformation of $Z$ in $T$ is a linear transformation, resulting in $T$ probability density function, defined by (7), and $T$ cumulative density function, defined by (8). The probability and cumulative density functions of $T$ random variable are demonstrated in Figures 5(a) and 5(b), respectively,

$$
\begin{aligned}
& f_{T}(t)= \begin{cases}0 & \text { if } t \leq \mathscr{C} \\
\frac{C_{1}}{8 \widehat{d}_{i} \hat{d}_{j} \varepsilon_{1} \varepsilon_{2}}\left[\left(\hat{d}_{j}+\varepsilon_{2} \widehat{d}_{j}\right)^{2}-\frac{\left(\widehat{d}_{i}-\varepsilon_{1} \widehat{d}_{i}\right)^{2}}{\left(t+C_{2}\right)^{2} C_{1}^{2}}\right] & \text { if } \mathscr{C} \leq t \leq \mathscr{B} \\
\frac{C_{1}}{8 \widehat{d}_{i} \widehat{d}_{j} \varepsilon_{1} \varepsilon_{2}}\left[\frac{\left(\hat{d}_{i}+\varepsilon_{1} \widehat{d}_{i}\right)^{2}}{\left(t+C_{2}\right)^{2} C_{1}^{2}}-\left(\widehat{d}_{j}-\varepsilon_{2} \widehat{d}_{j}\right)^{2}\right] & \text { if } \mathscr{B} \leq t \leq \mathscr{A} \\
0 & \text { if } t \geq \mathscr{A}\end{cases} \\
& F_{T}(t)= \begin{cases}0 & \text { if } t \leq \mathscr{C} \\
\frac{C_{1}}{8 \widehat{d}_{i} \hat{d}_{j} \varepsilon_{1} \varepsilon_{2}}\left[(t-\mathscr{C})\left(\widehat{d}_{j}+\varepsilon_{2} \widehat{d}_{j}\right)^{2}-\frac{\left(\widehat{d}_{i}-\varepsilon_{1} \widehat{d}_{i}\right)^{2}}{C_{1}^{2}}\left(\frac{1}{t+C_{2}}-\frac{1}{\mathscr{C}+C_{2}}\right)\right] & \text { if } \mathscr{C} \leq t \leq \mathscr{B} \\
\frac{C_{1}}{8 \widehat{d}_{i} \widehat{d}_{j} \varepsilon_{1} \varepsilon_{2}}\left[\frac{\left(\widehat{d}_{i}+\varepsilon_{1} \widehat{d}_{i}\right)^{2}}{C_{1}^{2}}\left(\frac{1}{\mathscr{B}+C_{2}}-\frac{1}{t+C_{2}}\right)-\left(\widehat{d}_{j}-\varepsilon_{2} \widehat{d}_{j}\right)^{2}(t-\mathscr{B})\right] & \text { if } \mathscr{B} \leq t \leq \mathscr{A} \\
1 & \text { if } t \geq \mathscr{A}\end{cases}
\end{aligned}
$$


where

$$
\begin{aligned}
& C_{1}=\sin \left(\alpha_{i j}\right) \\
& C_{2}=\operatorname{cotan}\left(\alpha_{i j}\right) \\
& \mathscr{A}=\frac{\widehat{d}_{i}+\varepsilon_{1} \widehat{d}_{i}}{\left(\widehat{d}_{j}-\varepsilon_{2} \widehat{d}_{j}\right) C_{1}}-C_{2} \\
& \mathscr{B}=\frac{\widehat{d}_{i}}{\widehat{d}_{j} C_{1}}-C_{2} \\
& \mathscr{C}=\frac{\widehat{d}_{i}-\varepsilon_{1} \widehat{d}_{i}}{\left(\widehat{d}_{j}+\varepsilon_{2} \widehat{d}_{j}\right) C_{1}}-C_{2}
\end{aligned}
$$

From the probability density function (7), we can define the error probability. From the two angles $\beta_{1}$ and $\beta_{2}$, which delimit the beam of a node and $B W$ that represents the beamwidth or aperture of the directional antenna, we have

$$
\begin{gathered}
\beta_{1}, \beta_{2} \in(0, \pi) \\
\beta_{1}+\beta_{2}=B W \\
\beta_{1}<\beta_{2}
\end{gathered}
$$

$$
\begin{aligned}
\operatorname{cotan}\left(\beta_{1}\right) & >\operatorname{cotan}\left(\beta_{2}\right) \\
t & =\operatorname{cotan}(\theta)
\end{aligned}
$$

An error occurs when the resulting angle from calculations made from the map is less than the lower beam limit, in this case $\beta_{1}$, or greater than the upper limit, $\beta_{2}$. Therefore, the probability of error is given by $P_{e}\left[\theta<\beta_{1}\right.$ or $\left.\theta>\beta_{2}\right]$, which can be rewritten as $P_{e}\left[t<\operatorname{cotan}\left(\beta_{2}\right)\right.$ or $\left.t>\operatorname{cotan}\left(\beta_{1}\right)\right]$. Then, we derive the error probability of the density function, as shown in (7).

The error probability given by (11) depends on various parameters, i.e., the distances $d_{i}$ and $d_{j}$, the angle $\alpha_{i j}$, the beamwidth $B W$, and the distance error values $\varepsilon$. In order to understand the behavior of the error probability, we have fixed the values of $d_{i}$ and $d_{j}$, so that $d_{i}>d_{j}$. Figure $6($ a) shows the error probability as function of the beamwidth for different values of the distance error. We can observe that the error probability decreases with the increase of the beamwidth and becomes almost constant for beamwidths larger than $30^{\circ}$. This latter observation is related to the fact that to increase the beamwidth does not alter the region covered by the beam that results in a decrease of the error probability. Conversely, Figure 6(b) shows the error probability as function of the distance error for different beamwidths. One can notice that for small values of the distance error the probability goes to zero for larger beamwidths.

$$
\begin{aligned}
& \text { if } \operatorname{cotan}\left(\beta_{2}\right)<\operatorname{cotan}\left(\beta_{1}\right)<\mathscr{C} \\
& \text { if } \operatorname{cotan}\left(\beta_{2}\right)<\mathscr{C}<\operatorname{cotan}\left(\beta_{1}\right)<\mathscr{B} \\
& \text { if } \operatorname{cotan}\left(\beta_{2}\right)<\mathscr{C}<\mathscr{B}<\operatorname{cotan}\left(\beta_{1}\right)<\mathscr{A} \\
& \text { if } \mathscr{C}<\operatorname{cotan}\left(\beta_{2}\right)<\operatorname{cotan}\left(\beta_{1}\right)<\mathscr{B} \\
& \text { if } \mathscr{C}<\operatorname{cotan}\left(\beta_{2}\right)<\mathscr{B}<\operatorname{cotan}\left(\beta_{1}\right)<\mathscr{A} \\
& \text { if } \mathscr{C}<\operatorname{cotan}\left(\beta_{2}\right)<\mathscr{B}<\mathscr{A}<\operatorname{cotan}\left(\beta_{1}\right) \\
& \text { if } \mathscr{B}<\operatorname{cotan}\left(\beta_{2}\right)<\operatorname{cotan}\left(\beta_{1}\right)<\mathscr{A} \\
& \text { if } \mathscr{B}<\operatorname{cotan}\left(\beta_{2}\right)<\mathscr{A}<\operatorname{cotan}\left(\beta_{1}\right) \\
& \text { if } \mathscr{A}<\operatorname{cotan}\left(\beta_{2}\right)<\operatorname{cotan}\left(\beta_{1}\right) \\
& \text { if } \operatorname{cotan}\left(\beta_{2}\right)<\mathscr{C}<\mathscr{B}<\mathscr{A}<\operatorname{cotan}\left(\beta_{1}\right)
\end{aligned}
$$

\section{Simulations and Results}

This section describes the simulation scenario used to compare the proposed protocol to another protocol from the literature called MDND [4]. The performance metrics evaluated are the beam hit rate (BHR) and the average of time spent to completely discover the network (ANDT, average network discovery time). The first metric corresponds to the fraction of all obtained angles that would result in a successful transmission among two nodes. The second metric consists in the time to execute all phases of the protocol plus the average time spent in transmissions considering that all nodes wish to transmit to all other nodes; i.e., each node has to execute the refinement beamforming with all other nodes. 


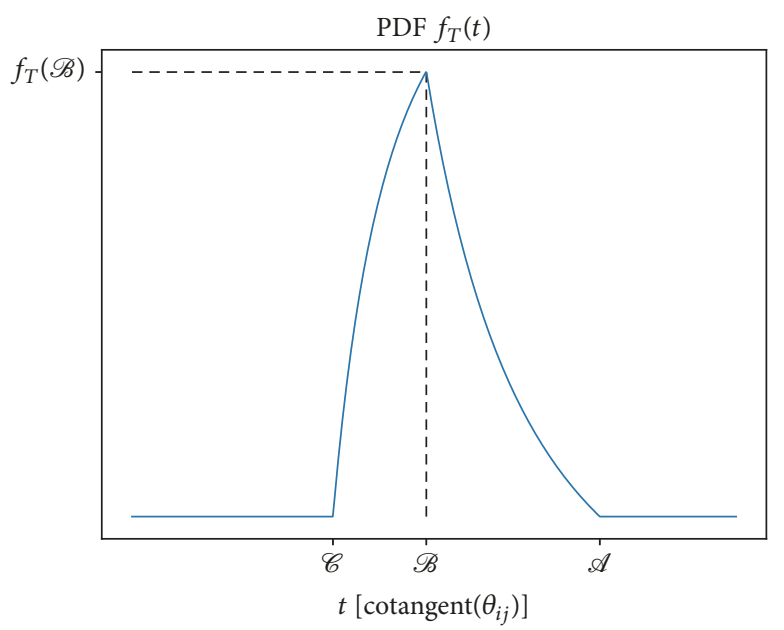

(a) Probability density function

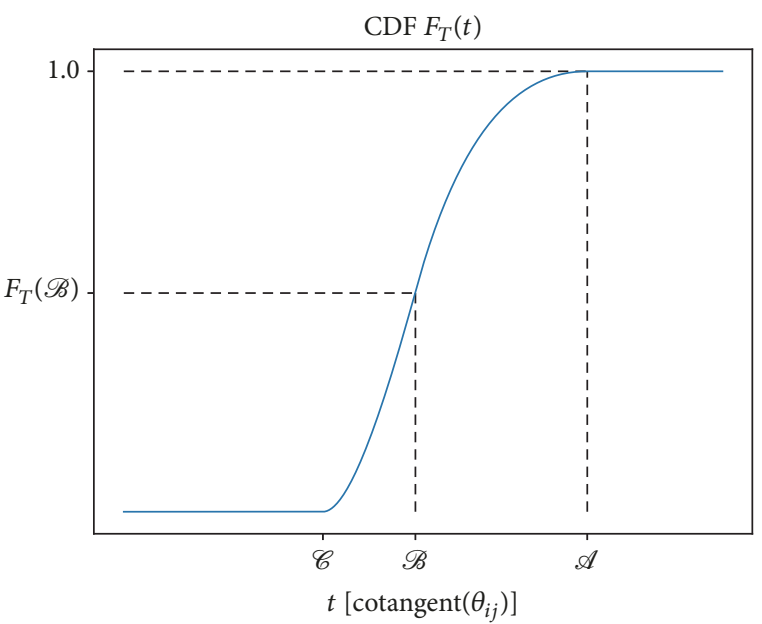

(b) Cumulative density function

FIGURE 5: Density functions of $T$ random variable.

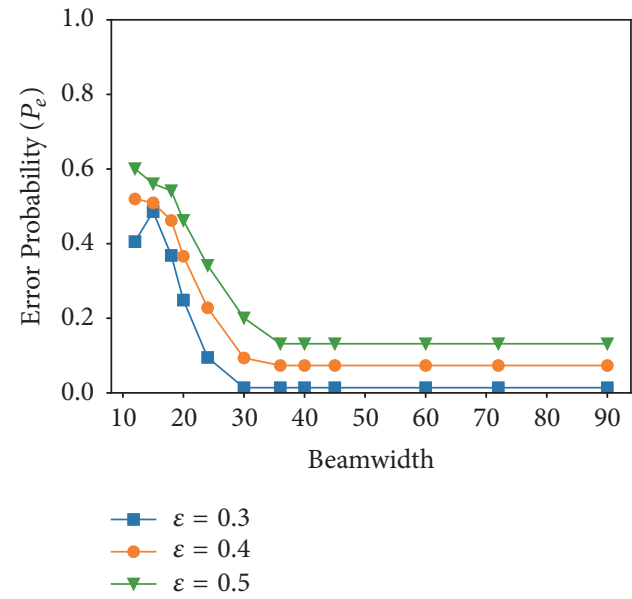

(a) Error probability versus beamwidth

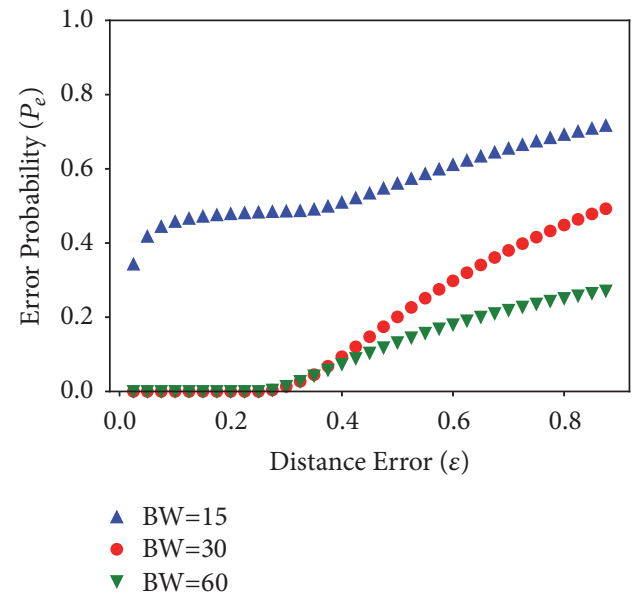

(b) Error probability versus distance error

FIGURE 6: Error probability fixing $d_{i}>d_{j}, \varepsilon_{1}=\varepsilon_{2}=\varepsilon$ and the angle $\alpha_{i j}$ remains static.

The simulation scenario consists in a $10 \mathrm{~m} \times 10 \mathrm{~m}$ room, where the nodes are randomly and uniformly distributed. Directional antennas are not steerable, but they are electronically switchable. The antenna model adopted is Idealized FlatTop Antenna System, which has been employed in some other works [11, 27]. The Idealized Flat-Top Antenna System gain, described by (12), increases as the beamwidth decreases. We also would like to emphasize that all nodes are stationary, due to the lack of mobility models that fit in the directional antennas and mmWave scenario and to maintain compatibility with previous works.

$$
G_{a}=10 \log \frac{2 \pi}{B_{w}}
$$

The simulator was implemented in Python and makes use of different mathematical libraries. The simulation consists on the following steps: (1) disposing the nodes in the area; (2) calculating the time to elect a leader and select the cluster leaders and corresponding regular nodes; (3) performing the beamforming within each cluster and among the cluster leaders; and (4) propagating the maps inside each cluster. Step (2) is calculated according to the time to exchange all messages needed by a distributed leader election algorithm and the assignment of the cluster leaders. Note that all these messages are exchanged in the control channel. The time spent in the step (3) corresponds to an exhaustive beamforming of the cluster leader with all its regular nodes and all other cluster leaders. Finally, the time spent in step (4) consists on the time spent to send via the control channel the final map to each node in the cluster.

Simulation results are averaged over 500 different runs by varying the parameters listed in Table 1 . When varying the number of sectors, we keep the number of nodes constant in 15. Moreover, when varying the number of nodes, we keep the nodes operating with 8 sectors. All curves show a 95\% confidence interval around the average. 
TABLE 1: Simulation parameters of CMPND and MDND protocols.

\begin{tabular}{|c|c|}
\hline Parameter & Value \\
\hline Phy Header Size & 16 Bytes \\
\hline Mac Header Size & 34 Bytes \\
\hline Ack Length & 31 Bytes \\
\hline Sector Sweep Frame Length & 26 Bytes \\
\hline Sector Sweep Feedback Frame Length & 31 Bytes \\
\hline Map Frame Length & $(10 *$ Nodes $)$ Bytes \\
\hline Data Frame Length & 1024 Bytes \\
\hline Beam Refinement Frame Length & 1024 Bytes \\
\hline RSV_REQ Frame Length & 20 Bytes \\
\hline RSV_RES Frame Length & 14 Bytes \\
\hline SIFS (Control Band) & $10 \mu \mathrm{S}$ \\
\hline Slot & $9 \mu \mathrm{S}$ \\
\hline DIFS & $28 \mu \mathrm{S}$ \\
\hline SIFS (Data Band) & $3 \mu \mathrm{S}$ \\
\hline SBIFS & $1 \mu \mathrm{S}$ \\
\hline Ack Timeout (Control Band) & $100 \mu \mathrm{S}$ \\
\hline Ack Timeout (Data Band) & $300 \mu \mathrm{S}$ \\
\hline Control Band transfer rate & $54 \mathrm{Mbps}$ \\
\hline Data Band transfer rate & 1 Gbps \\
\hline Number of nodes & {$[15,30,45,60]$} \\
\hline Number of Antenna's Sectors & {$[4,8,16,32]$} \\
\hline
\end{tabular}

4.1. Beam Hit Rate. The beam hit rate (BHR) metric shows how much the inference of node position from the map is accurate. As explained before, the more accurate is this inference, the smaller is the time needed to the beam refinement before a successful transmission takes place. In order to measure the gain obtained by the clusterization, we have included in the simulations the MPND mechanism (CMPND without clusterization) by using only one cluster leader. Additionally, two different refinement configurations are examined. The first configuration to be tested infers the angle in a three-beam interval, which led to a less accurate estimation that demands a longer refinement. The other configuration does the same estimation in the interval of one beam, more accurate but also prone to cause more errors in estimation.

The beam hit rate for network with $K$ devices is described by $(13)$. Where $\mathscr{I}(i, j)$ represents an indicator function which is equal to 1 if the transmission from node $i$ to node $j$ was successfully done and equal to 0 otherwise.

$$
B H R=\frac{\sum_{i} \sum_{j \neq i} \mathscr{I}(i, j)}{K \times(K-1)}
$$

As shown in Figures 7(a) and 7(b) there is only a difference of $4 \%$ by the use of clusters. This allows us to state that errors are included in the clustering process. This increase in the error is due to the corrections of angles made by the cluster leaders and the nodes when transmitting, as explained in Section 3.1. The transmission angle for a node in another cluster must first be calculated by the cluster leader of the transmitter's cluster and then recalculated by the transmitter itself. The first operation is because the cluster leaders share maps between them and these shared maps need to be fixed before being distributed inside their clusters. The second operation is the calculation of the transmitter from the map provided by the cluster leader. This chain of calculations inserts more errors than a direct operation, as Figures 8(a) and 8(b) allow us to infer. There is an influence of beamwidth reduction on beam hit rate and the protocol performs better when no clustering is applied, due the imprecision inserted by the clustering mechanism, as aforementioned.

4.2. Average Neighbor Discovery Time. The average neighbor discovery time (ANDT) estimates the time spent from the beginning of neighbor discovery procedure to the end of one transmission. Equation (14) expresses mathematically the average neighbor discovery time.

$$
A N D T=T_{\text {overhead }}+\frac{\sum_{1}^{n} T_{m s g}}{n}
$$

where $T_{\text {overhead }}$ represents the time taken on neighbor discovery control phase, beamforming, and any other operation related to node positioning and location. With the purpose of validating the discovery and realizing a beam refinement, $n$ messages are sent through the network. If one message is 


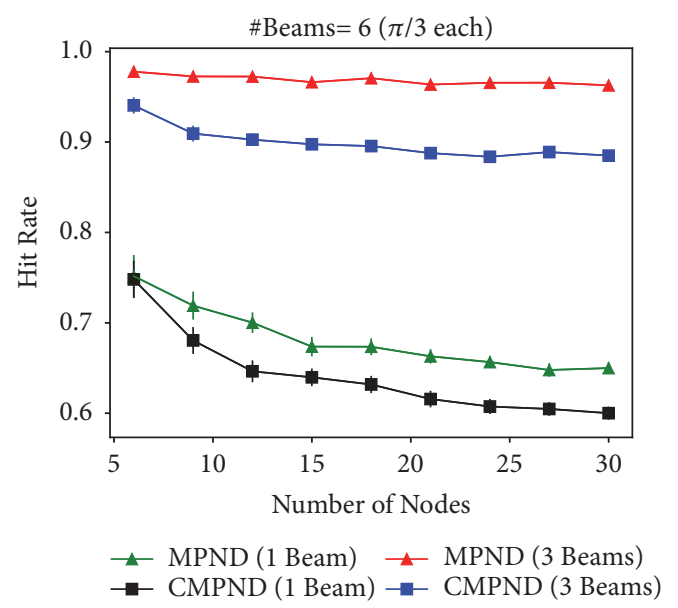

(a)

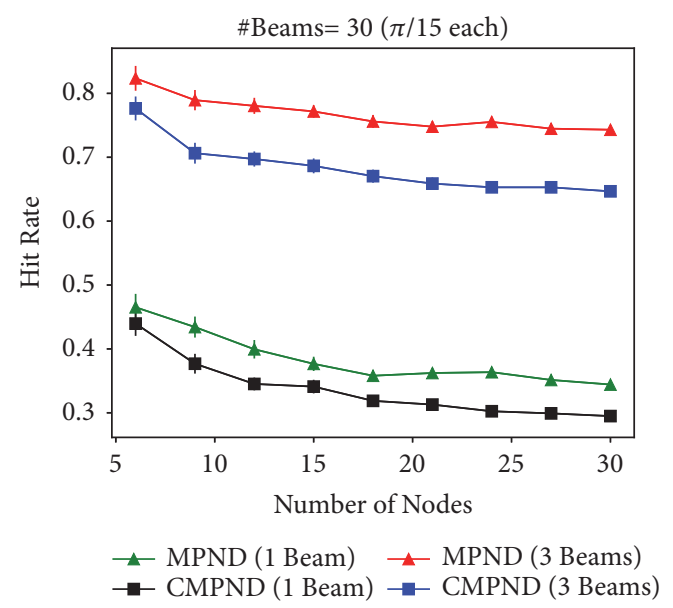

(b)

FIGURE 7: Beam hit rate (BHR) under different number of nodes composing the network, varying the number of beams in (a) 6 and (b) 30.

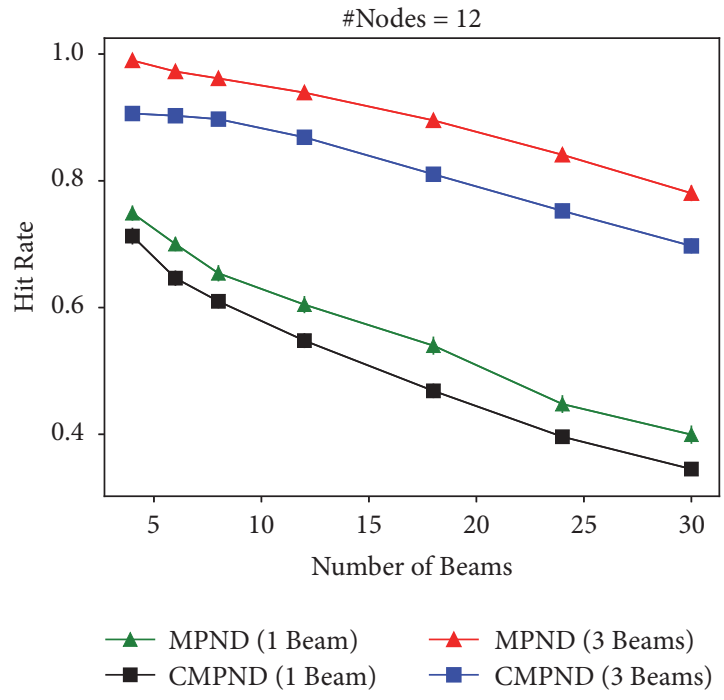

(a)

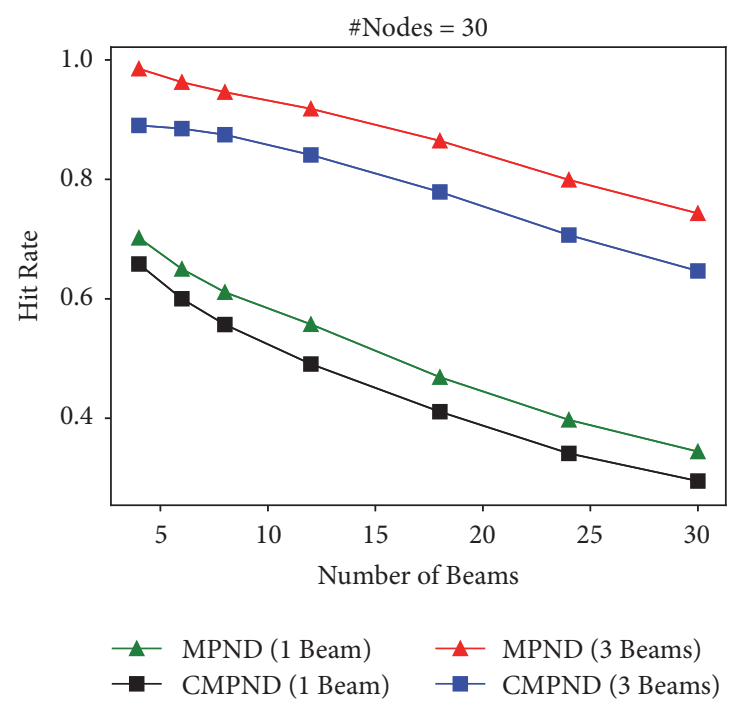

(b)

FIGURE 8: BHR under different number of beams configurations, varying the number of nodes in (a) 12 and (b) 30.

not successfully transmitted, the discovery must be revised to adjust the angle. One node can detect an error by waiting for an ack timeout without receiving anything. Thus, each angle error increases the time to transmit a message. Finally, we sum the overhead with the average time to transmit a message, $T_{m s g}$.

In Figures 9(a) and 9(b) we observe a reduction in average time when comparing CMPND to MDND. The reduced amount of control messages sent by nodes running CMPND resulted in a considerable decrease on neighbor discovery time.

On the other hand, the impact of increasing the number of beams is notable on CMPND, as we can see on Figures 10 (a) and 10(b). Augmenting the number of beams, which means the reduction of the beamwidth, makes the angle error critical and leads to more time spent on angle corrections till the nodes were able to transmit. Despite overperforming MDND, the average neighbor discovery time grows faster when increasing the number of beams on CMPND.

\section{Conclusion}

In this work, we propose a neighbor discovery protocol for ad hoc millimeter wave networks denominated CMPND. It uses a multiband approach for the clusterization of the nodes and the construction of a map with the nodes positioning in terms of beamforming. The propagation of this map by the cluster leaders allows the nodes to build their own neighbor localization map without the need of an exhaustive beamforming. Also, we derive an analytical model for the 


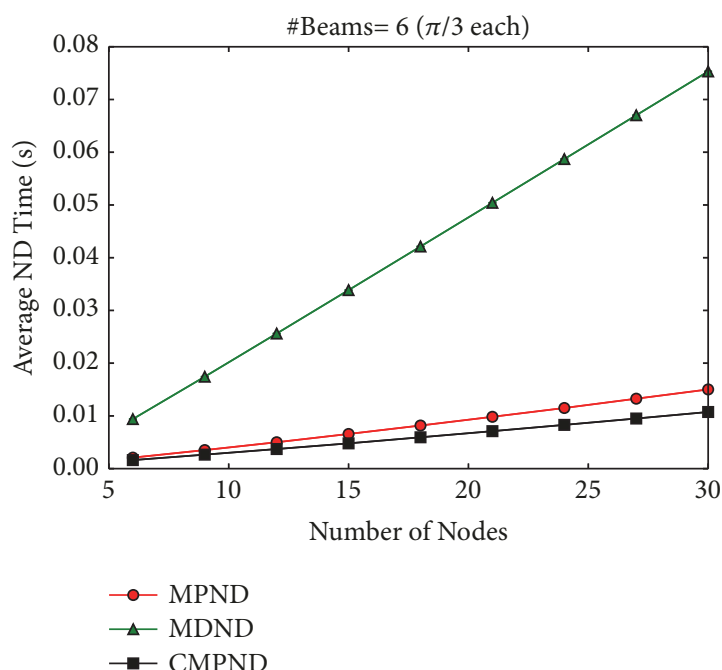

(a)

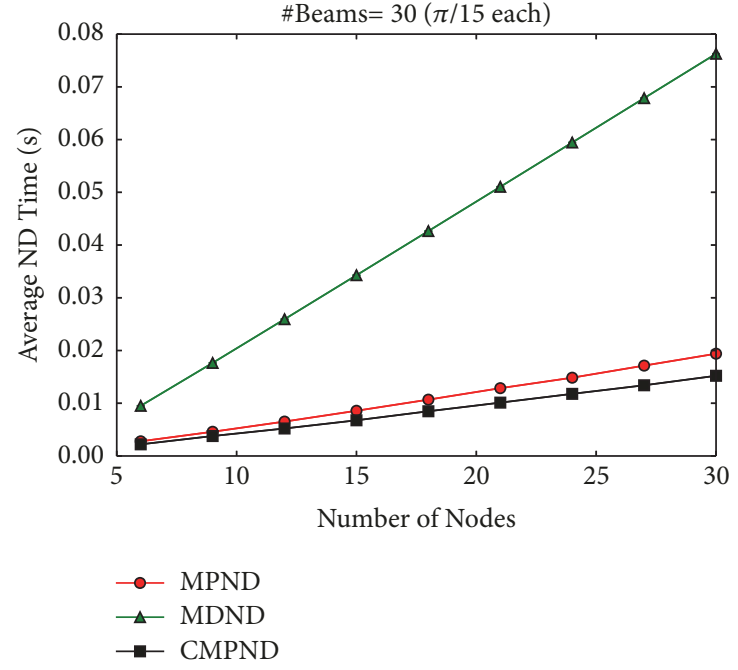

(b)

FIGURE 9: Average neighbor discovery time (ANDT) under different number of nodes, varying the number of beams (a) 6 and (b) 30.

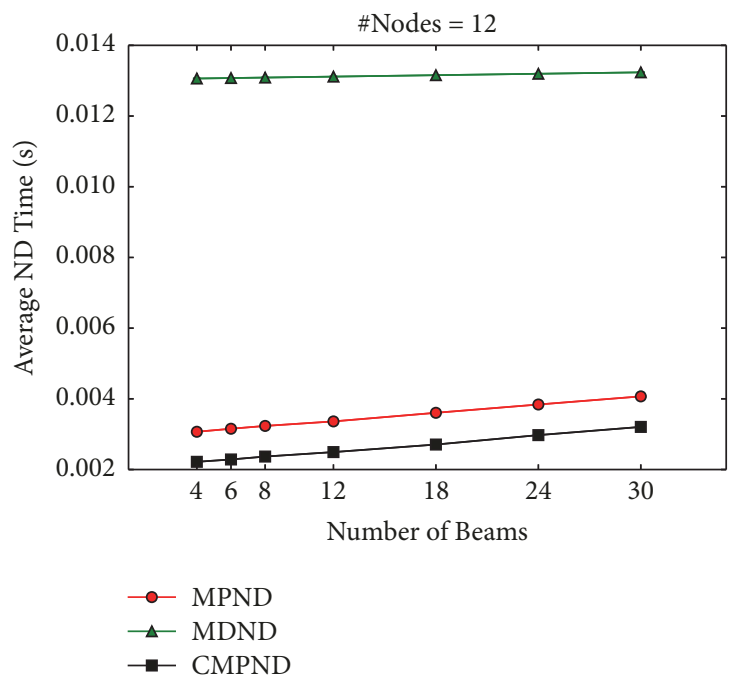

(a)

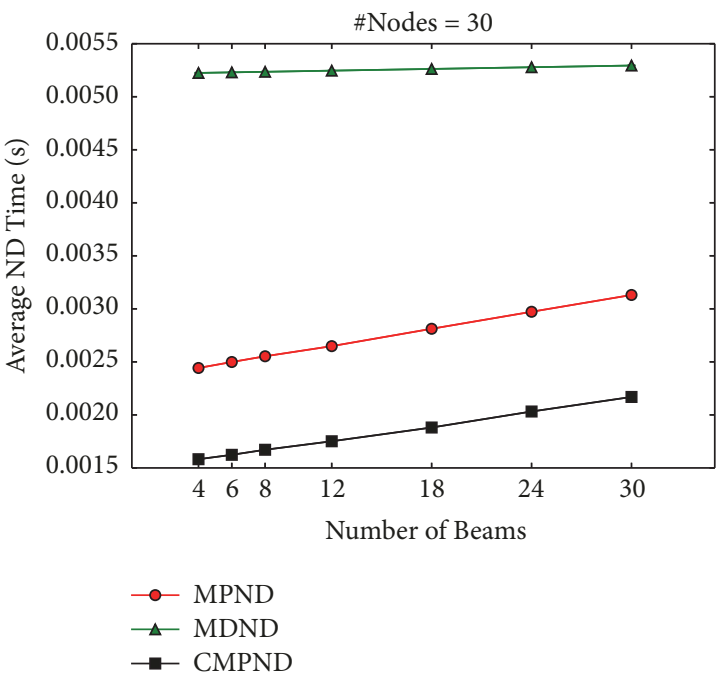

(b)

FIGURE 10: ANDT under different number of beams, varying the number of nodes in (a) 12 and (b) 30 .

error probability in finding the beam that achieves the highest SNR using this approach.

By comparing CMPND with other existing protocol, we found that it reduces the time spent in the neighbor discovery by half in a network composed by 15 nodes, regardless of the inaccuracy increase that the clustering process leads. This is due to the fact that it drastically decreases both the number of beamforming types and the access to the shared CSMA-CA control channel.

In the future, we intend to exploit the mathematical model that describes the error probability in order to have some insights on the definition of new strategies for neighbor discovery. In addition, we plan to investigate the possibility of achieving spatial reuse in the directional communications by leveraging the global map. Another perspective of this work is to enlarge the neighbor discovery for multihop millimeter wave networks and advance in cluster leader selection using auxiliary metrics like computational power and power consumption.

\section{Data Availability}

No data were used to support this study.

\section{Conflicts of Interest}

The authors declare that they have no conflicts of interest.

\section{References}

[1] Y. Niu, Y. Li, D. Jin, L. Su, and A. V. Vasilakos, "A survey of millimeter wave communications (mmWave) for 5G: opportunities 
and challenges," Wireless Networks, vol. 21, no. 8, pp. 2657-2676, 2015.

[2] G. M. Ölçer, Z. Genç, and E. Onur, "Smart neighbor scanning with directional antennas in $60 \mathrm{GHz}$ indoor networks," in Proceedings of the 21st Annual IEEE International Symposium on Personal, Indoor and Mobile Radio Communications, pp. 23932398, IEEE, 2010.

[3] S. Sur, V. Venkateswaran, X. Zhang, and P. Ramanathan, " $60 \mathrm{Ghz}$ indoor networking through flexible beams: a linklevel profiling," in Proceedings of the International Conference on Measurement and Modeling of Computer Systems, SIGMETRICS '15, pp. 71-84, ACM, New York, NY, USA, 2015.

[4] H. Park, Y. Kim, T. Song, and S. Pack, "Multiband directional neighbor discovery in self-organized mmWave Ad Hoc networks," IEEE Transactions on Vehicular Technology, vol. 64, no. 3, pp. 1143-1155, 2015.

[5] M. Agiwal, A. Roy, and N. Saxena, "Next generation 5G wireless networks: a comprehensive survey," IEEE Communications Surveys and Tutorials, vol. 18, no. 3, pp. 1617-1655, 2016.

[6] J. Qiao, X. S. Shen, J. W. Mark, Q. Shen, Y. He, and L. Lei, "Enabling device-to-device communications in millimeterwave 5G cellular networks," IEEE Communications Magazine, vol. 53, no. 1, pp. 209-215, 2015.

[7] S. Rangan, T. S. Rappaport, and E. Erkip, "Millimeter-wave cellular wireless networks: potentials and challenges," Proceedings of the IEEE, vol. 102, no. 3, pp. 366-385, 2014.

[8] J. Ning, T.-S. Kim, S. V. Krishnamurthy, and C. Cordeiro, "Directional neighbor discovery in $60 \mathrm{GHz}$ indoor wireless networks," Performance Evaluation, vol. 68, no. 9, pp. 897-915, 2011.

[9] IEEE 802.11ad, IEEE Standard for Information technologyTelecommunications and information exchange between systems Local and metropolitan area networks-Specific requirements Part 11: Wireless LAN Medium Access Control (MAC) and physical Layer (PHY) Specifications Amendment 3: Enhancements for Very High throughput in the $60 \mathrm{GHz}$ Band. IEEE Std 802.11ad-2012 (Amendment to IEEE Std 802.11-2012, as amended by IEEE Std 802.11ae-2012 and IEEE Std 802.11aa2012), 1-628, 2012.

[10] T. Nitsche, C. Cordeiro, A. B. Flores, E. W. Knightly, E. Perahia, and J. C. Widmer, "IEEE 802.11ad: Directional $60 \mathrm{GHz}$ communication for multi-gigabit-per-second Wi-Fi," IEEE Communications Magazine, vol. 52, no. 12, pp. 132-141, 2014.

[11] X. An, R. V. Prasad, and I. Niemegeers, "Impact of antenna pattern and link model on directional neighbor discovery in $60 \mathrm{GHz}$ networks," IEEE Transactions on Wireless Communications, vol. 10, no. 5, pp. 1435-1447, 2011.

[12] H. Assasa and J. Widmer, "Extending the IEEE 802.11ad model: scheduled access, spatial reuse, clustering, and relaying," in Proceedings of the Workshop on Ns-3, WNS3 '17, pp. 39-46, ACM, New York, NY, USA, 2017.

[13] R. Amiri and H. Mehrpouyan, "Self-organizing mm wave networks: a power allocation scheme based on machine learning," in Proceedings of the 11th Global Symposium on Millimeter Waves (GSMM '18), pp. 1-4, IEEE, 2018.

[14] B. Soleimani and M. Sabbaghian, "Cluster-based resource allocation and user association in mmwave femtocell networks," IEEE Transactions on Communications, p. 1, 2018.

[15] H. Xu, T. Zhao, S. Zhu, D. Lv, and J. Zhao, "Agglomerative group scheduling for mmwave massive MIMO under hybrid beamforming architecture," in Proceedings of the 18th International
Conference on Communication Technology (ICCT '18), pp. 347351, IEEE, 2018.

[16] Y. Wang and G. De Veciana, "Dense indoor mmWave wearable networks: managing interference and scalable MAC," in Proceedings of the 14th International Symposium on Modeling and Optimization in Mobile, Ad Hoc, and Wireless Networks (WiOpt '16), pp. 1-8, IEEE, 2016.

[17] G. H. Sim, T. Nitsche, and J. C. Widmer, "Addressing MAC layer inefficiency and deafness of IEEE802.1lad millimeter wave networks using a multi-band approach," in Proceedings of the 27th Annual International Symposium on Personal, Indoor, and Mobile Radio Communications (PIMRC '16), pp. 1-6, IEEE, 2016.

[18] D. Da Silva Brilhante and J. F. De Rezende, "Multiband neighbor discovery protocols for millimeter waves Ad Hoc networks," in Proceedings of the 25th International Conference on Telecommunications, ICT '18, pp. 511-515, IEEE, 2018.

[19] G. Jakllari, L. Wenjie, and S. V. Krishnamurthy, "An integrated neighbor discovery and MAC protocol for ad hoc networks using directional antennas," IEEE Transactions on Wireless Communications, vol. 6, no. 3, pp. 1114-1124, 2007.

[20] L. Chen, Y. Li, and A. V. Vasilakos, "Oblivious neighbor discovery for wireless devices with directional antennas," in Proceedings of the 35th Annual IEEE International Conference on Computer Communications, INFOCOM '16, pp. 1-9, IEEE, 2016.

[21] V. C. Barbosa, An Introduction to Distributed Algorithms, vol. 1, Mit Press, 1996.

[22] A. Olivier, G. Bielsa, I. Tejado, M. Zorzi, J. Widmer, and P. Casari, "Lightweight indoor localization for $60-\mathrm{GHz}$ millimeter wave systems," in Proceedings of the 13th Annual IEEE International Conference on Sensing, Communication, and Networking (SECON ;16), pp. 1-9, IEEE, 2016.

[23] J. Palacios, P. Casari, and J. Widmer, "JADE: zero-knowledge device localization and environment mapping for millimeter wave systems," in Proceedings of the IEEE Conference on Computer Communications, INFOCOM '17, pp. 1-9, IEEE, 2017.

[24] N. Maletic, V. Sark, J. Gutierrez, and E. Grass, "Device localization using mmWave ranging with sub-6-assisted angle of arrival estimation," in Proceedings of the International Symposium on Broadband Multimedia Systems and Broadcasting (BMSB '18), pp. 1-6, IEEE, 2018.

[25] H. Xu, V. Kukshya, and T. S. Rappaport, "Spatial and temporal characteristics of 60-GHZ indoor channels," IEEE Journal on Selected Areas in Communications, vol. 20, no. 3, pp. 620-630, 2002.

[26] H. El-Sayed, G. Athanasiou, and C. Fischione, "Evaluation of localization methods in millimeter-wave wireless systems," in Proceedings of the 19th International Workshop on Computer Aided Modeling and Design of Communication Links and Networks (CAMAD '14), pp. 345-349, IEEE, 2014.

[27] S. Vasudevan, J. Kurose, and D. Towsley, "On neighbor discovery in wireless networks with directional antennas," in Proceedings of the 24th Annual Joint Conference of the IEEE Computer and Communications Societies, vol. 4, pp. 2502-2512, IEEE, 2005. 


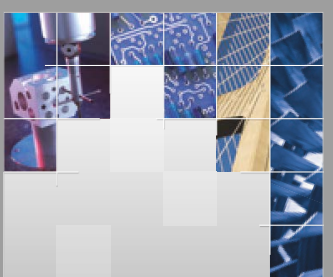

\section{Enfincering}
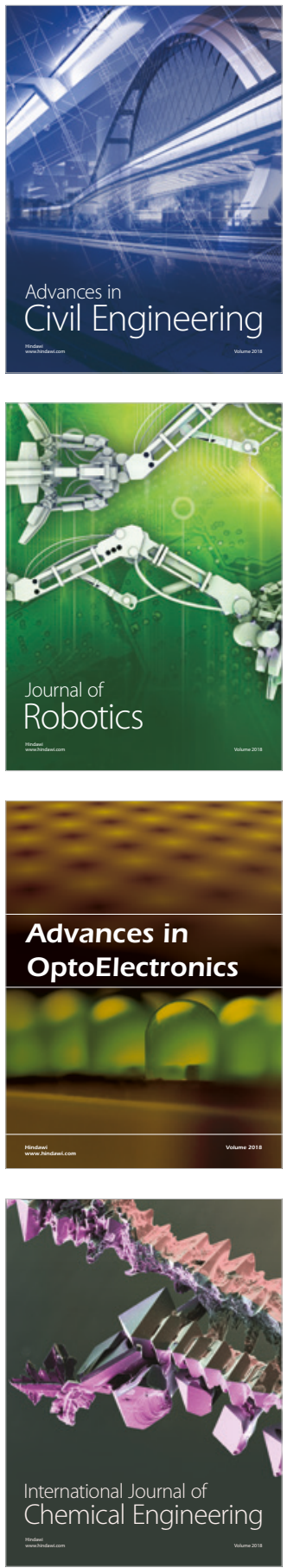

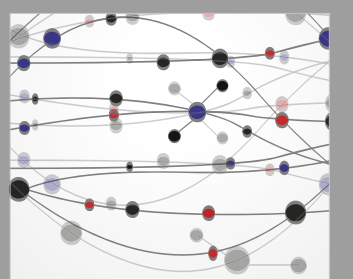

\section{Rotating \\ Machinery}

The Scientific World Journal

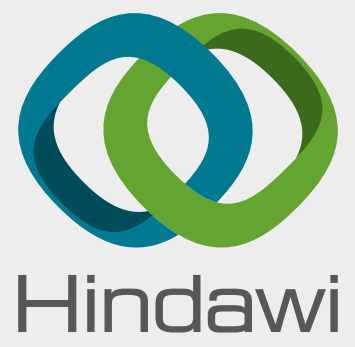

Submit your manuscripts at

www.hindawi.com
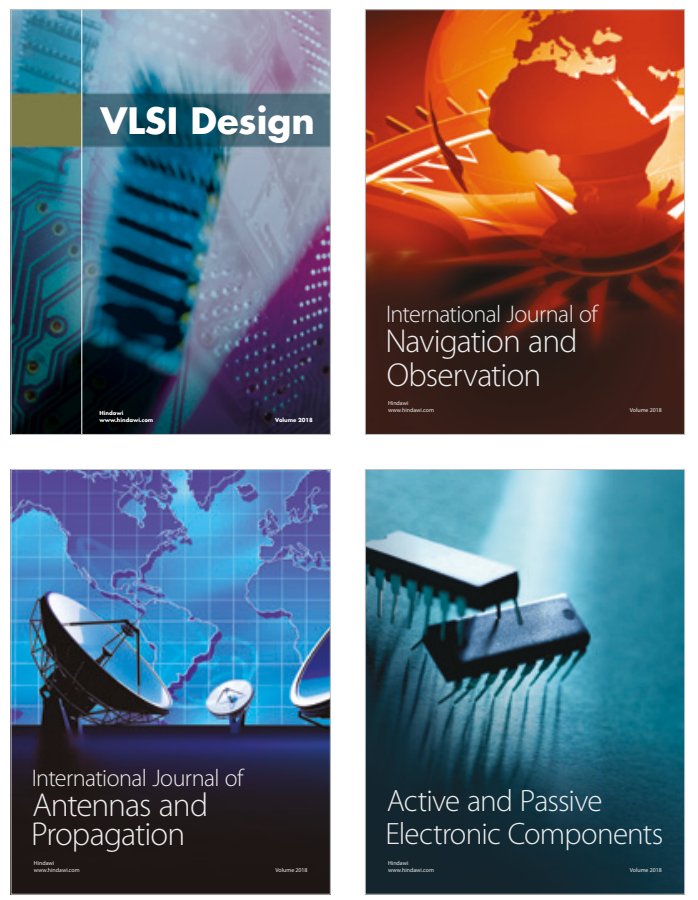
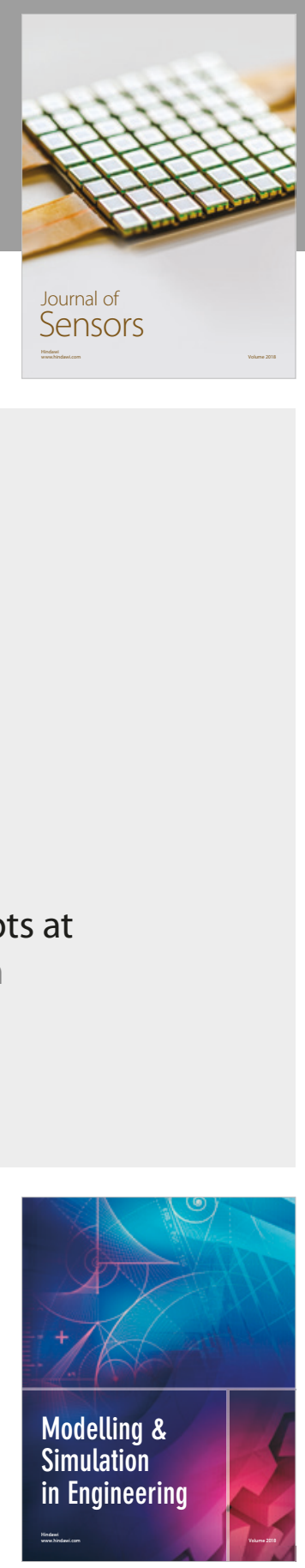

\section{Advances \\ Multimedia}
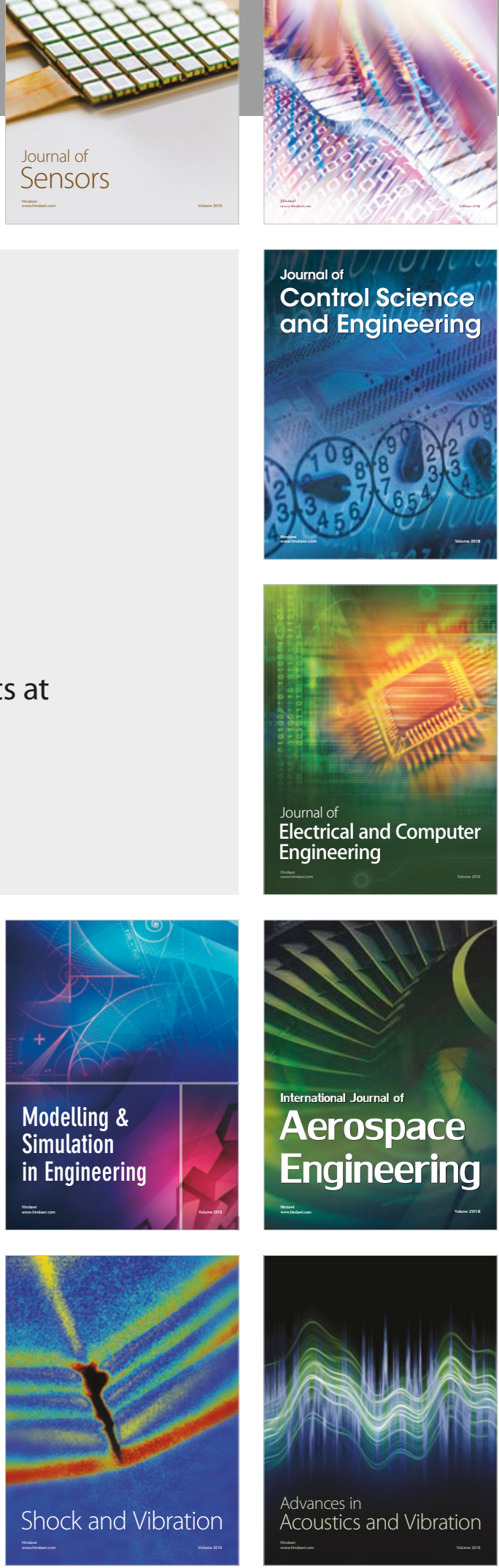